Check for updates

Cite this: RSC Adv., 2019, 9, 5480

Received 19th November 2018

Accepted 7th February 2019

DOI: $10.1039 / c 8 r a 09491 h$

rsc.li/rsc-advances

\section{Tussah silkmoth pupae improve anti-tumor properties of Cordyceps militaris (L.) Link by increasing the levels of major metabolite cordycepin $\dagger$}

\author{
Zhixin Wen, Xingfan Du, iD * Nan Meng, ${ }^{*}$ Yajie Li, Rui Mi, Xuejun Li, Yongxin Sun, \\ Shuhui Ma and Shuying Li
}

\begin{abstract}
Silkworms have been reported to promote the growth and production of the stromata of C. militaris (L.) Link as a parasite insect medium and may improve its metabolites. The effects of Tussah silkmoth pupae (TG group) and rice (RG group) on the metabolic profile of C. militaris (L.) Link were compared by metabolomics. Meanwhile, the profile of natural $C$. sinensis (NG group) was also analyzed. The functions of these metabolites from different groups and cordycepin were tested using breast cancer cells and an animal model. 292 metabolites were detected, including 51, 31 and 23 unique metabolites from the TG, RG and NG groups, respectively. The level of 3-deoxyadenosine (cordycepin with anti-tumor activity) was highest in the TG group. Tussah silkmoth pupae induced the biosynthesis of cordycepin and unsaturated fatty acids, which may be beneficial in the prevention of breast cancer. The TG group and cordycepin had significant inhibitory activities on breast cancer cells and in animal models when compared with the two other groups. Tussah silkmoth pupae improved the metabolic profile of $C$. militaris (L.) Link, which has more pharmaceutical metabolites than C. sinensis.
\end{abstract}

\section{Introduction}

Cordyceps sinensis, a kind of traditional Chinese medicine, exerts many health-promoting properties. The phagocytic activities of the mononuclear phagocytes increase during bacterial infection. C. sinensis can improve phagocytic activities of the phagocytes by increasing the levels of interferon-gamma, interleukin (IL)-12, and tumor necrosis factor- $\alpha$, but not IL-1 $\beta$, IL-6, or IL-8. ${ }^{1}$ The extracts of $C$. sinensis had anti-metastatic activities by preventing the hepatocyte growth factor-mediated tumor invasiveness of melanoma cells. ${ }^{2}$ C. sinensis could reduce carbon tetrachloride-induced liver inflammation and fibrosis by activating natural killer cells and reversing artisanal small-scale gold mining-1-mediated depletion of hepatic nature killer cells. ${ }^{3} C$. sinensis has been reported to improve the healthrelated quality of life of the patients with asthma and inflammatory symptoms. ${ }^{4}$ A $C$. sinensis exopolysaccharide-conjugated selenium nanoparticles exerted high-antioxidant for $\mathrm{O}^{2-}$ and the radical cation 2,2'-azinobis-(3-ethylbenzothiazoline-6-sulfonate). ${ }^{5}$ C. sinensis can be used as raw materials of some functional foods to improve and regulate the sub-health status of

Dalian Biotechnology Institute, Liaoning Academy of Agricultural Sciences, Shida Street No. 2, Dalian 116024, China.E-mail: dxfan68@126.com; mona0408@163. com; Fax: +8641184790092; Tel: +8641184790092

$\dagger$ Electronic supplementary information (ESI) available. See DOI: 10.1039/c8ra09491h humans. Nevertheless, the production of the $C$. sinensis is still low and the species cannot be cultured in a large scale.

Cordyceps militaris (L.) Link, another Cordyceps species, has been widely cultured manually and also has numerous potential medicinal properties including antitumor activity.,7 A monocarbohydrate, xylitol, isolated from C. militaris, could induce apoptotic death of malignant melanoma but not in normal human fibroblasts, suggesting that $C$. militaris extract was a potential antitumor agent. ${ }^{7}$ Silkworm has been reported to promote the growth and production of stromata of C. militaris (L.) Link as parasite insect media. ${ }^{8}$ However, their metabolites have never been compared between C. sinensis and C. militaris (L.) Link. Thus, it is necessary to compare their metabolites to exploit the pharmaceutical values.

Metabolomics is an emerging and rapidly developed technology that can help us to comprehensively understand the metabolite profiles of the two species. Therefore, to obtain more information on metabolites, metabolome was used to compare the difference for the metabolite profiles of two species. Meanwhile, the effects of the different media Tussah silkmoth pupae and rice on the metabolite profile of $C$. militaris (L.) Link was also investigated.

\section{Materials and methods}

\subsection{Materials}

C. militaris (L.) Link and C. sinensis was purchased from Dalian Qiyun Health pharmacy (Dalian, China). Tussah silkmoth 
pupae was purchased from Guizhou Sericulture Institute (Zunyi, China). Rice was purchased from local supermarket (Dalian, China).

\subsection{The culture of $C$. militaris (L.) Link}

C. militaris (L.) Link was inoculated in a $10 \mathrm{~mL}$ liquid medium containing $0.3 \%$ potassium dihydrogen phosphate, $0.15 \%$ magnesium sulfate and $2 \%$ rice, at $25{ }^{\circ} \mathrm{C}$ for $7 \mathrm{~d}$. One-mL of the seeding $C$. militaris (L.) Link were inoculated into sterilized rice medium (RG group, $100 \mathrm{~g}$ ) at $18-22{ }^{\circ} \mathrm{C}$ with $12 \mathrm{~h}$ light daily and $90 \%$ humidity for $50 \mathrm{~d}$. One-mL seeding species were inoculated into Tussah silkmoth pupae (TG group, $100 \mathrm{~g}$ total) and cultured with above situation.

\subsection{Preparation of Cordyceps extracts}

The mycelium of C. militaris (L.) Link (about $40 \mathrm{~g}$ and $50 \mathrm{~g}$ biomass from rice and Tussah silkmoth pupae, respectively) and natural Cordyceps sinensis (NG group, $50 \mathrm{~g}$ ) were collected, frozen under liquid nitrogen, and ground with a pestle and a mortar. The powder was extracted with $400 \mathrm{~mL}, 500 \mathrm{~mL}$ and $500 \mathrm{~mL} 80 \% \mathrm{MeOH}$ for $2 \mathrm{~d}$ under reflux, respectively. The collected extracts were centrifuged at $12000 \mathrm{~g} \times 10 \mathrm{~min}$ and filtered with a $10 \mathrm{kDa}$ cut-off filter (Millipore Corp., Bedford, MA, USA). Finally, all the extracts were concentrated to dry in a rotary evaporator at $50{ }^{\circ} \mathrm{C}$.

One-milligram of purified cordycepin and standard, were mixed with $100 \mathrm{mg}$ potassium bromide, ground finely, compressed, and then were scanned at wave number 4000$400 \mathrm{~cm}^{-1}$. The IR spectra were compared between purified cordycepin and standard.

\subsection{GC/MS-based metabolomic analyses}

Thirty-mg samples were transferred to a $1.5 \mathrm{~mL}$ sterilized Eppendorf tube. $360 \mu \mathrm{L}$ of cold methanol and $40 \mu \mathrm{L}$ of 2 -chloroL-phenylalanine $\left(0.3 \mathrm{mg} \mathrm{mL}{ }^{-1}\right)$ were added to the tube, and stored at $-80{ }^{\circ} \mathrm{C}$ for $2 \mathrm{~min}$. The mixtures were ultrasonicated at $25{ }^{\circ} \mathrm{C}$ for half an hour. $200 \mu \mathrm{L}$ of chloroform was added to the samples, and the mixtures were vortexed, and $400 \mu \mathrm{L}$ water was added. Samples were centrifuged at $12000 \mathrm{~g}, 4{ }^{\circ} \mathrm{C}$ for $10 \mathrm{~min}$. $500 \mu \mathrm{L}$ of supernatant was dried in a glass vial in a freeze concentration centrifugal dryer (Chennai L\&T Corporate, Tamil Nadu, India). $80 \mu \mathrm{L}$ of $15 \mathrm{mg} \mathrm{mL} \mathrm{m}^{-1}$ methoxylamine hydrochloride in pyridine was subsequently added. The resultant mixture was vortexed vigorously for $2 \mathrm{~min}$ and incubated at $37{ }^{\circ} \mathrm{C}$ for $90 \mathrm{~min} .80 \mu \mathrm{L}$ of BSTFA (with 1\% TMCS) and $20 \mu \mathrm{L} n$-hexane was added into the mixture, which was vortexed vigorously for $2 \mathrm{~min}$ and then evaporated at $70{ }^{\circ} \mathrm{C}$ for $60 \mathrm{~min}$. The samples were placed at $25{ }^{\circ} \mathrm{C}$ for $30 \mathrm{~min}$ before GC-MS analysis. The dried samples were analyzed on an Agilent 7890B gas chromatography system coupled to an Agilent 5977A MSD system (Agilent, CA, USA). A DB-5MS fused-silica capillary column $(30 \mathrm{~m} \times$ $0.25 \mathrm{~mm} \times 0.25 \mu \mathrm{m}$, Agilent $\mathrm{J} \& \mathrm{~W}$ Scientific, Folsom, CA, USA) was utilized to separate the derivatives. Helium (>99.999\%) was used as the carrier gas at a constant flow rate of $1 \mathrm{~mL} \mathrm{~min} \mathrm{~m}^{-1}$ through the column. The injector temperature was maintained at $260^{\circ} \mathrm{C}$. Injection volume was $1 \mu \mathrm{L}$ by split mode (split ratio
$4: 1$ ). The initial oven temperature was $60^{\circ} \mathrm{C}$, ramped to $125^{\circ} \mathrm{C}$ at a rate of $8{ }^{\circ} \mathrm{C} \mathrm{min}$, to $210{ }^{\circ} \mathrm{C}$ at a rate of $4{ }^{\circ} \mathrm{C} \mathrm{min}$, to $270{ }^{\circ} \mathrm{C}$ at a rate of $5{ }^{\circ} \mathrm{C} \mathrm{min}$, to $305{ }^{\circ} \mathrm{C}$ at a rate of $10{ }^{\circ} \mathrm{C} \mathrm{min}$, and finally held at $305{ }^{\circ} \mathrm{C}$ for $3 \mathrm{~min}$. The temperature of MS quadrupole, and ion source (electron impact) was set at 150 and $230^{\circ} \mathrm{C}$, respectively. The collision energy was $70 \mathrm{eV}$. Mass data was acquired in a full-scan mode $(\mathrm{m} / \mathrm{z} 50-450)$, and the solvent delay time was set to $5 \mathrm{~min}$. The QCs were injected at regular intervals (every 6 samples) throughout the analytical run to provide a set of data from which repeatability can be assessed.

\subsection{Fatty acid analysis}

Fatty acid is one of the important active ingredients in $C$. sinensis. ${ }^{9}$ The fatty acids were analyzed in three groups. Above samples were placed in $15 \mathrm{~mL}$ centrifuge tube, and following chemicals were added, including $2 \mathrm{~mL}$ of $5 \%$ hydrochloric acid and methanol $(1: 1, \mathrm{v} / \mathrm{v}), 3 \mathrm{~mL}$ chloroform-methanol, and methyl decanoate (internal standard solution, $10 \mu \mathrm{g} \mathrm{mL}^{-1}$ ) 100 $\mu \mathrm{L}$. The mixture was incubated in a water bath at $85^{\circ} \mathrm{C}$ for $1 \mathrm{~h}$. One-mL $n$-hexane was added and shaken for $2 \mathrm{~min}$. The final solution was filtered through $0.45 \mu \mathrm{m}$ microporous membrane and stored at $4{ }^{\circ} \mathrm{C}$. The GC-MS method was established to simultaneously determine the tetradecanoic acid (C14.0), pentadecanoic acid (C15.0), palmitic acid (C16.0), palmitoleic acid (C16.1), heptadecanoic acid (C17.0), heptadecenoic acid (C17.1), octadecanoic acid (C18.0), methyl oleate (C18.1N9C), linoleic acid (C18.2N6C), arachidic acid (C20.0), arachidonic acid (C20.1), arachidonic acid (C20.2), eicosatrienoic acid (C20.3N6), twenty-one carbonic acid (C21.0), behenic acid (C22.0), twenty-three carbonic acid (C23.0), and wood taric acid (C24.0). The contents of fatty acids were compared among three groups. Mixed fatty acid methyl ester (batch number: CDAA251019M, purity: >99\%) and methyl decanoate (batch number: S2257B21589, purity: >99\%) were purchased from $\mathrm{Nu}-$ Chek Company (Elysian, MN, USA).

The following chromatographic condition was used: a Thermo Scientific TG-5MS capillary column $(30 \mathrm{~m} \times 0.25 \mathrm{~mm}$ $\times 0.25 \mu \mathrm{m}$ ); carrier gas, nitrogen; flow rate, $1.2 \mathrm{~mL} \mathrm{~min}^{-1}$; inlet temperature, $290{ }^{\circ} \mathrm{C}$; splitless injection; valve opening time, $1 \mathrm{~min}$; injection volume, $1 \mu \mathrm{L}$. The standards were precisely weighed, placed in a $10 \mathrm{~mL}$ volumetric flask, and diluted with $n$ hexane to prepare a series of mixed reference solutions with fatty acid methyl ester concentrations of 10, 50, 100, 300, and $500 \mu \mathrm{g} \mathrm{mL} \mathrm{m}^{-1}$, respectively. One $\mu \mathrm{L}$ of above mixed control solutions was measured according to the above conditions. Linear regression was performed with the fatty acid methyl ester mass concentration $\left(x, \mu \mathrm{g} \mathrm{mL}{ }^{-1}\right)$ as the abscissa and the ion signal relative intensity $(y)$ as the ordinate. The regression equation and linear range were shown in Table 1 . The limit of quantitation was obtained when the signal-to-noise ratio was $10: 1$, the detection limit was obtained when the signal-to-noise ratio was $3: 1$.

The following mass spectrometry situation was performed: electrospray ionization source; ion source temperature/ transmission line temperature, $280{ }^{\circ} \mathrm{C}$; column temperature, initial temperature $80{ }^{\circ} \mathrm{C}$, programmed temperature $80^{\circ} \mathrm{C}$ for 
Table 1 Regression equations, linear ranges, limits of quantification and limits of detection in fatty acid analysis

\begin{tabular}{|c|c|c|c|c|c|}
\hline Fatty acid & Regression equation & $r$ & Linear range, $\mu \mathrm{g} \mathrm{mL}^{-1}$ & $\begin{array}{l}\text { Limit of } \\
\text { quantitation, } \mathrm{ng} \mathrm{mL} \mathrm{m}^{-1}\end{array}$ & $\begin{array}{l}\text { Limit of } \\
\text { detection, } \mathrm{g} \mathrm{mL}^{-1}\end{array}$ \\
\hline C14.0 & $y=0.082 x+0.521$ & 0.9998 & $1.420-44.530$ & 64.29 & 19.34 \\
\hline C16.0 & $y=0.169 x+1.453$ & 0.9982 & $3.146-85.852$ & 33.96 & 10.29 \\
\hline C16.1 & $y=0.122 x+0.444$ & 0.9987 & $1.664-62.444$ & 80.21 & 24.06 \\
\hline C17.0 & $y=0.129 x+0.483$ & 0.9995 & $1.773-64.983$ & 87.98 & 26.39 \\
\hline C18.1N9C & $y=0.095 x+0.489$ & 0.9996 & $1.439-47.984$ & 41.67 & 12.50 \\
\hline C18.2N6C & $y=0.133 x+0.408$ & 0.9996 & $1.738-66.909$ & 99.67 & 29.90 \\
\hline C20.0 & $y=0.188 x+0.206$ & 0.9995 & $2.086-94.208$ & 122.45 & 36.73 \\
\hline C20.1 & $y=0.089 x+0.467$ & 0.9994 & $1.356-44.966$ & 118.11 & 35.43 \\
\hline $\mathrm{C} 20.2$ & $y=0.113 x+0.315$ & 0.9997 & $1.444-56.814$ & 129.87 & 38.96 \\
\hline C20.3N6 & $y=0.104 x+0.336$ & 0.9998 & $1.375-52.335$ & 108.69 & 32.61 \\
\hline
\end{tabular}

$1 \mathrm{~min}$, at a rate of $10{ }^{\circ} \mathrm{C} \mathrm{min}^{-1}$ to $200{ }^{\circ} \mathrm{C}$; increasing to $250{ }^{\circ} \mathrm{C}$ at $5{ }^{\circ} \mathrm{C} \min ^{-1}$; ramping to $270{ }^{\circ} \mathrm{C}$ at $2{ }^{\circ} \mathrm{C} \mathrm{min}^{-1}$ for $3 \mathrm{~min}$; ionization voltage, $70 \mathrm{eV}$; solvent delay time, $5 \mathrm{~min}$ and scan mass range, $m / z 30-400$.

\subsection{Screening for different metabolites}

The multi-dimensional analysis and single-dimensional analysis were used to screen the differential metabolites between groups. The screening standard was VIP value $>1$ of a consensus orthogonal partial least squares discriminant analysis (OPLSDA) model. ${ }^{10}$ The difference for the changes of metabolites was compared in the fold change (FC) between two groups. The fold change was the ratio of the average content of the difference metabolites in both groups. FC $>1$ was up-regulation, and $\mathrm{FC}<1$ was down-regulation.

\subsection{Heat-map analysis}

In order to show the difference of the up-regulation and downregulation of metabolites, the heat-map quantitative analysis of the shared metabolites was performed among three groups. $\mathrm{R}$ language (version 3.1.1) was used to draw the cluster heat map.

\subsection{Network analysis}

A string database, which is a functionally relevant database that searches for systems of known and predicted molecule interactions, ${ }^{11}$ was used to perform network analysis uses. This interaction includes both direct and indirect physical interactions between molecules. The network figure was drawn by combing top ten paths of the KEGG (Kyoto encyclopedia of genes and genomes) databases and tools. ${ }^{12}$

\subsection{Anti-breast-tumor test}

This study was performed in strict accordance with the NIH guidelines for the care and use of laboratory animals (NIH Publication no. 85-23 Rev. 1985) and was approved by the
Institutional Animal Care and Use Committee of Liaoning Academy of Agricultural Science (Shenyang, China). Human breast cancer cell lines (MCF-7) were stored in our lab; 3 week BALB/c mice (16-18 g) were purchased from Dalian Medical University Experimental Animal Center (Dalian, China). RPMI1640 medium was purchased from GIBCO Company (NY, USA). Fetal bovine serum was purchased from Lanzhou Minhai Biological Engineering Co., Ltd (Lanzhou, China). Human breast cancer cells (MCF-7) were removed from liquid nitrogen at $-196{ }^{\circ} \mathrm{C}$, routinely resuscitated, and cultured in RPMI-1640 medium ( $\mathrm{pH} 7.2-7.4$ ) supplemented with 10\% fetal bovine serum at $37{ }^{\circ} \mathrm{C}, 5 \% \mathrm{CO}_{2}$ incubator. The cells were grown for 3 days and then sub-cultured. MCF-7 cells were treated with different components and cordycepin for further 72 hour culture. The cells divided into 6 groups: control group, $20 \mu \mathrm{L}$ $\mathrm{L}^{-1}$ normal saline (CG group); $200 \mathrm{mg} \mathrm{L}^{-1}$ extracts of natural Cordyceps sinensis (NG group); $200 \mathrm{mg} \mathrm{L}^{-1}$ extracts of Cordyceps militaris (L.) Link cultured on rice (RG group); $200 \mathrm{mg} \mathrm{\textrm {L } ^ { - 1 }}$ extracts of Cordyceps militaris (L.) Link cultured on Tussah silkmoth pupae (TG group); $30 \mathrm{mg} \mathrm{L}^{-1}$ cordycepin (LG group); and $90 \mathrm{mg} \mathrm{L}^{-1}$ cordycepin ( $\left.\mathrm{kg} \mathrm{d}\right)^{-1}$ (HG group). Morphological changes of cells were observed under an inverted microscope (Olympus, Japan). The ultrastructural changes of cells were observed under transmission electron microscope JEM-1010 (JEOL, Japan).

Under sterile conditions, sarcoma-180 ascites tumor-bearing mice were inoculated for 10 days to disinfect the abdominal skin of the animal. Five-mL disposable sterile syringe was used to penetrate the abdominal cavity to absorb the tumor cells. In ascites, the ascites was placed in a sterile Erlenmeyer flask, placed on an ice box, and the ascites was diluted with physiological saline to adjust the tumor cell concentration to $1 \times 10^{7}$ $\mathrm{mL}^{-1}$.

$0.2 \mathrm{~mL}$ tumor cell suspension was inoculated subcutaneously into the right forelimb axilla of BALB/c mouse (the number of tumor cells was about $2 \times 10^{6} \mathrm{~mL}^{-1}$ ). The 


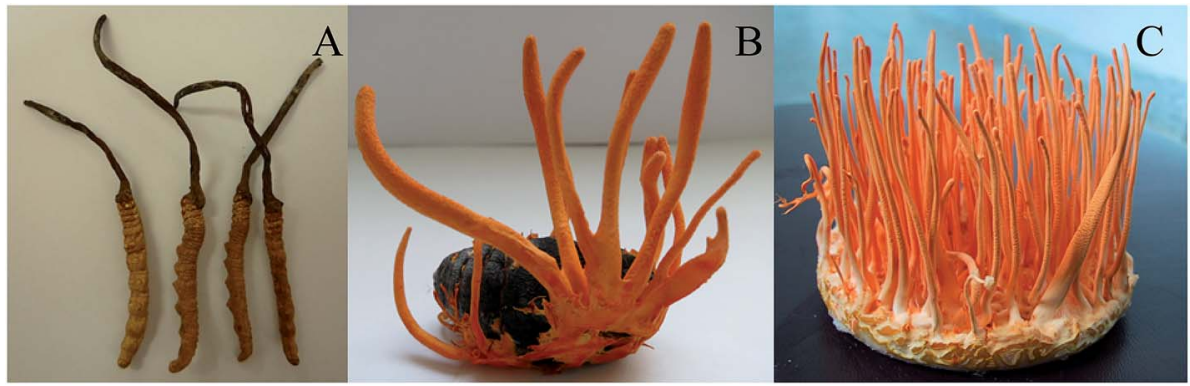

Fig. 1 The comparison of the mycelial morphology between C. militaris (L.) Link and C. sinensis. (A) Cordyceps militaris (L.) Link cultured on Tussah silkmoth pupae. (B) Cordyceps militaris (L.) Link cultured on rice. (C) Cordyceps sinensis.

experiment was divided into 6 groups, 10 mice in each group, and the number of male and female was equal. Tumor-bearing mice control group, $20 \mu \mathrm{L}(\mathrm{kg} \mathrm{d})^{-1}$ normal saline (CG group); $200 \mathrm{mg}(\mathrm{kg} \mathrm{d})^{-1}$ extracts of natural Cordyceps sinensis (NG group); $200 \mathrm{mg}\left(\mathrm{kg} \mathrm{d}^{-1}\right.$ extracts of Cordyceps militaris (L.) Link cultured on rice (RG group); $200 \mathrm{mg}(\mathrm{kg} \mathrm{d})^{-1}$ extracts of Cordyceps militaris (L.) Link cultured on Tussah silkmoth pupae (TG group); the extract of low-dose cordycepin $30 \mathrm{mg}(\mathrm{kg} \mathrm{d})^{-1}$ (LG group); and high-dose cordycepin $90 \mathrm{mg}$ ( $\mathrm{kg} \mathrm{d})^{-1}$ (HG group). Feeding regular feed and free drinking water during the experimental period, the tumor recombination was calculated for 10 days, and the mice were sacrificed the next day, and the tumor was weighed.

\subsection{MTT assay}

MTT assay was performed to determine the effect of cordycepin on the growth of MCF-7 cells according to a previous report. ${ }^{13}$ MCF-7 cells were treated with different concentration of cordycepin. The cells were then seeded in a 96-well plate and further cultivated for $3 \mathrm{~d}$ before MTT assay.

\subsection{Statistical methods}

Data were expressed as mean values \pm S.D. All data were analyzed by variance analysis via SPSS 20 software, and $P<0.05$ was considered significant.

\section{Results}

3.1. The comparison of the mycelial morphology between $C$. militaris (L.) Link and C. sinensis

C. sinensis and C. militaris belong to ascomycete of the Ascomycetes subfamily, but they are not a same genus. For TG group, carcass was oval with $1.5-2 \mathrm{~cm}$ long and 5-10 mm wide and the color was purplish purple; the mycelial was $4-12 \mathrm{~cm}$ in length, and 2-4 $\mathrm{mm}$ in width and the color was orange (Fig. 1A). For RG group, mycelial 2-8 cm long, and 1-2 mm wide color, and the color was orange (Fig. 1B). For the NG group, the worm
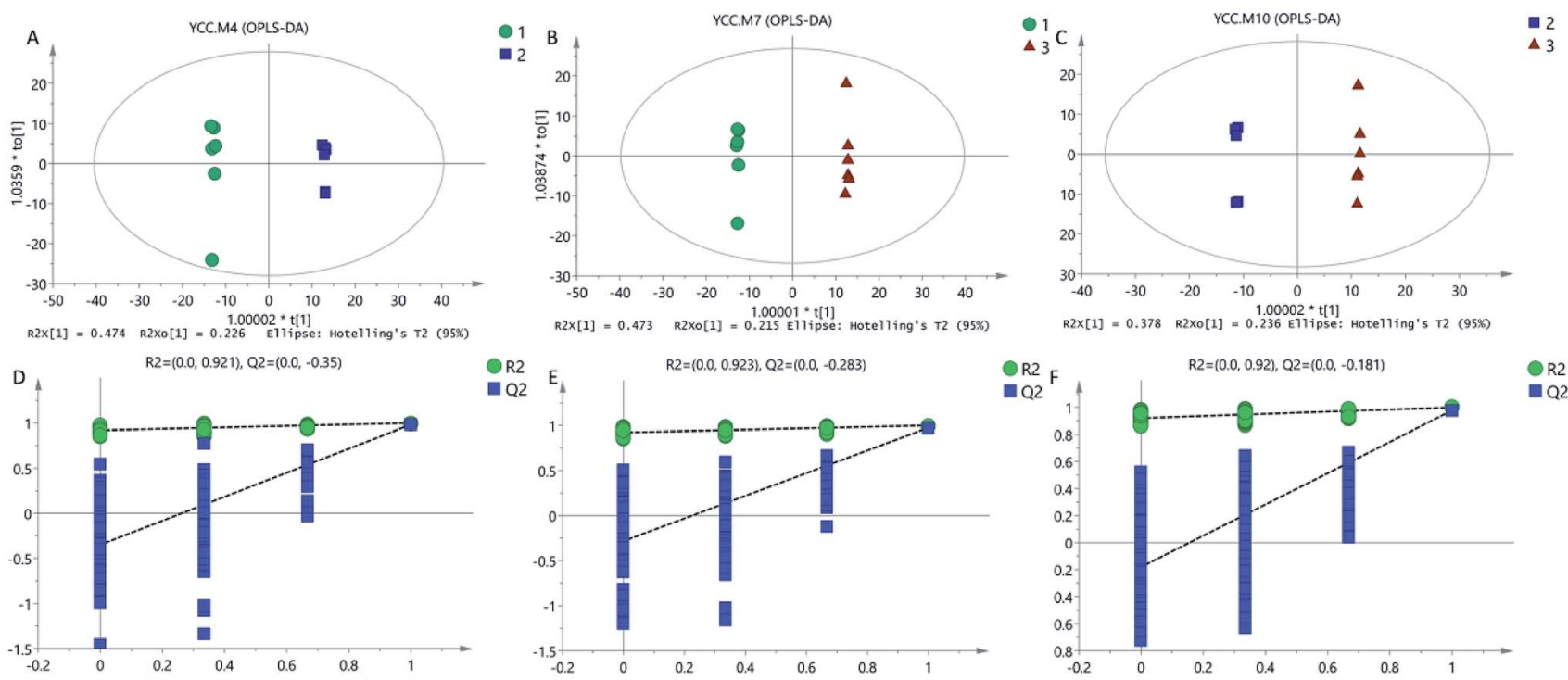

Fig. 2 OPLS-DA score plots and model validation among different groups. (A) OPLS-DA score plots of TG and RG. (B) OPLS-DA score plots of TG and NG. (C) OPLS-DA score plots of RG and NG. (D) OPLS-DA model validation of TG and RG. (E) OPLS-DA model validation of TG and NG. (F) OPLS-DA model validation of RG and NG. TG, Cordyceps militaris (L.) Link cultured on Tussah silkmoth pupae. RG, C. militaris (L.) Link cultured on rice. NG, C. sinensis. 


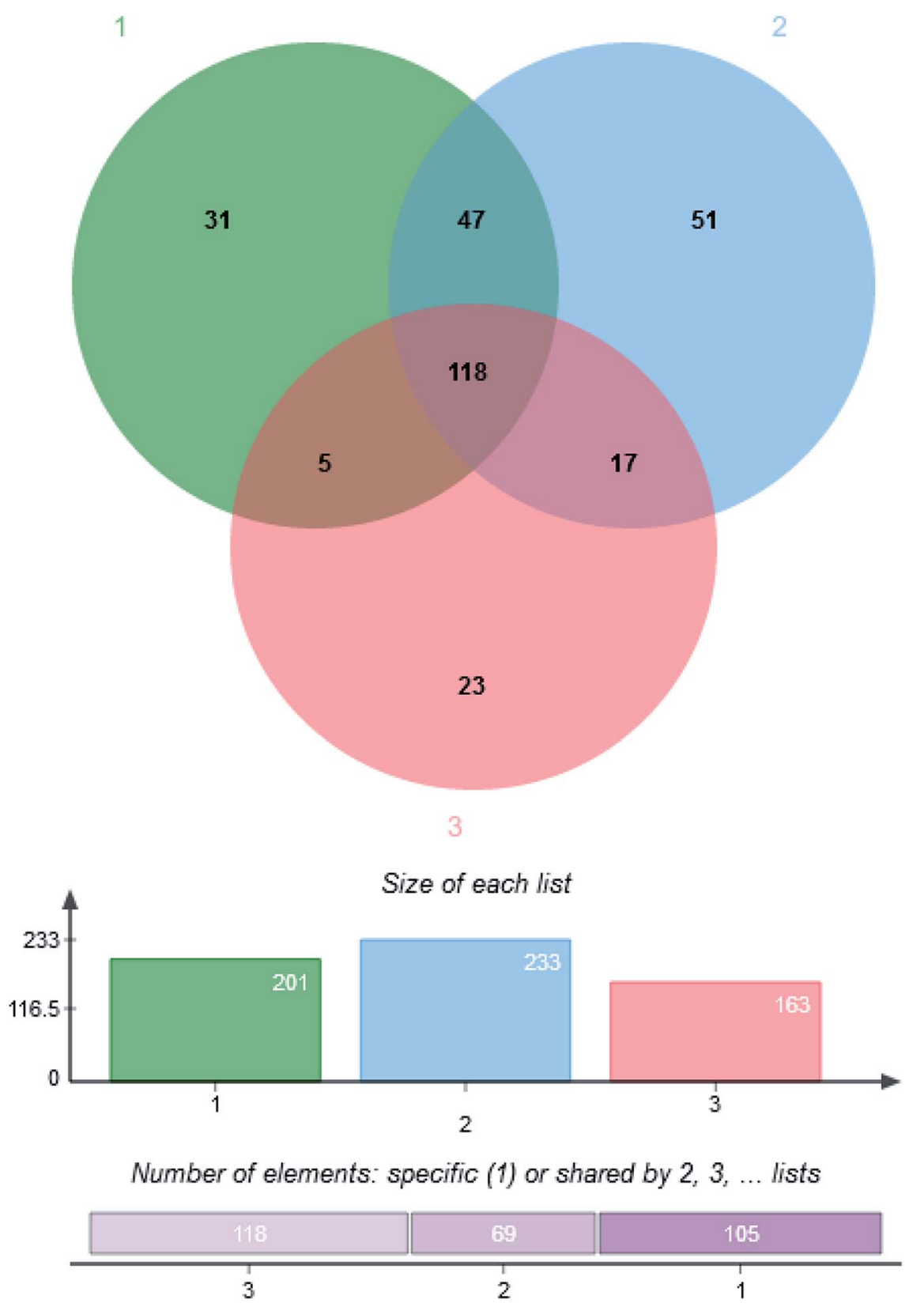

Fig. 3 Venn diagram of metabolite distribution among three groups. (1) Cordyceps militaris (L.) Link cultured on rice. (2) Cordyceps militaris (L.) Link cultured on Tussah silkmoth pupae. (3) Cordyceps sinensis.

was 3-5 cm long and 3-10 $\mathrm{mm}$ wide, and the color was brownish-yellow; C. sinensis grew from the head of the worm, and the color was brown or tan. The mycelial was $4-10 \mathrm{~cm}$ long and $1.54 \mathrm{~mm}$ thick.

\subsection{The comparison of metabolite constituents among different groups}

A multivariate analysis method was performed to discriminate the ions that contributed to the classification of different samples. OPLS-DA was employed for classification analyses and there was significant variation for Cordyceps militaris (L.) Link cultured on between Tussah silkmoth pupae and rice (Fig. 2A), between C. militaris (L.) Link cultured on Tussah silkmoth pupae and C. sinensis (Fig. 2B), and between C. militaris (L.) Link cultured on rice and $C$. sinensis (Fig. 2C). In the validated model, the slope coefficient of $R^{2}(0.0,0.921)$ and $Q^{2}(0.0,-0.350)$ (Fig. 2D), $R^{2}(0.0,0.923)$ and $Q^{2}(0.0,0.283)$ (Fig. 2E), and $R^{2}(0.0$, $0.920)$ and $Q^{2}(0.0,-0.181)$ (Fig. $\left.2 \mathrm{~F}\right)$, indicated that the established model was fit for analysis of different metabolite constituents among different groups.

\subsection{C. militaris (L.) Link has more metabolites than $C$. sinensis}

GC-MS data were extracted by using XCMS online metabolomics and the metabolites were matched. 292 kinds of metabolites were detected in the mycelium of $C$. militaris (L.) Link and $C$. 
(A)

sinensis, including 118 metabolites ( 8 alcohols, 47 acids, 3 alkanes, 15 sugar, 7 esters, 2 ketones, 14 amino acids, 2 amines, 3 adenosines and 17 other substances) shared by three groups, and 51, 31 and 23 unique metabolites from TG, CG and NG groups, respectively (Fig. 3).

\subsection{Analysis of metabolite profiles among the three groups}

Among the detected metabolites, there were 90 upregulated and 79 downregulated metabolites in the TG group when compared with the RG group (Fig. 4A). There were 102 upregulated and 66 downregulated metabolites in the TG group when compared with the NG group (Fig. 4B). There were 97 upregulated and 42 downregulated metabolites in the RG group when compared with the NG group (Fig. 4C). 3-Deoxyadenosine was produced at the highest level in TG group and has been reported to inhibit tumor proliferation and induces tumor apoptosis ${ }^{14}$. KEGG analysis showed that there were 48,6 and 9 unique metabolite pathways in TG, RG and NG groups, respectively. Among these pathways, the pathway involving with insulin secretion and biosynthesis of unsaturated fatty acids were induced in TG when compared with other two groups (Table S1†). Metabolome revealed that Tussah silkmoth pupae improved the metabolic profile C. militaris (L.) Link when compared those cultured on rice medium (Fig. 4). C. militaris (L.) Link had more metabolites than C. sinensis. Additionally, some potential pharmaceutical products such as 3-deoxygenadenosine, hesperitin, 3-hydroxyflavone and glycerol were up-regulated in the TG group, carnitine and alpha-tocopherol were up-regulated in the RG group, and gentiobiose and tartaric acid were upregulated in the NG group.

\subsection{Metabolic network analysis}

The comparison between TG and RG indicated that the metabolites pathways involving with sucrose, spermidine, glycerone, xanthosine, ribitol and naphthalene were induced (Fig. 5A). The comparison between TG and NG indicated that the metabolites pathways involving with sucrose, spermidine, xanthine, maltotriose, alpha-tocopherol, orthophosphate, 3.4dihydroxymandelate, 3.4-dihydroxybenzoate, pyridoxine, $\mathrm{N}$ acetylserotonin and naphthalene were induced (Fig. 5B). The comparison between RG and NG indicated that the metabolites pathways involving with spermidine and lipoate were induced (Fig. 5C).

\subsection{Tussah silkmoth pupae increased the contents of unsaturated fatty acids in C. militaris (L.) Link}

Table 2 showed that there were 16 kinds of fatty acids were shared among three groups. The content of oleic acid 

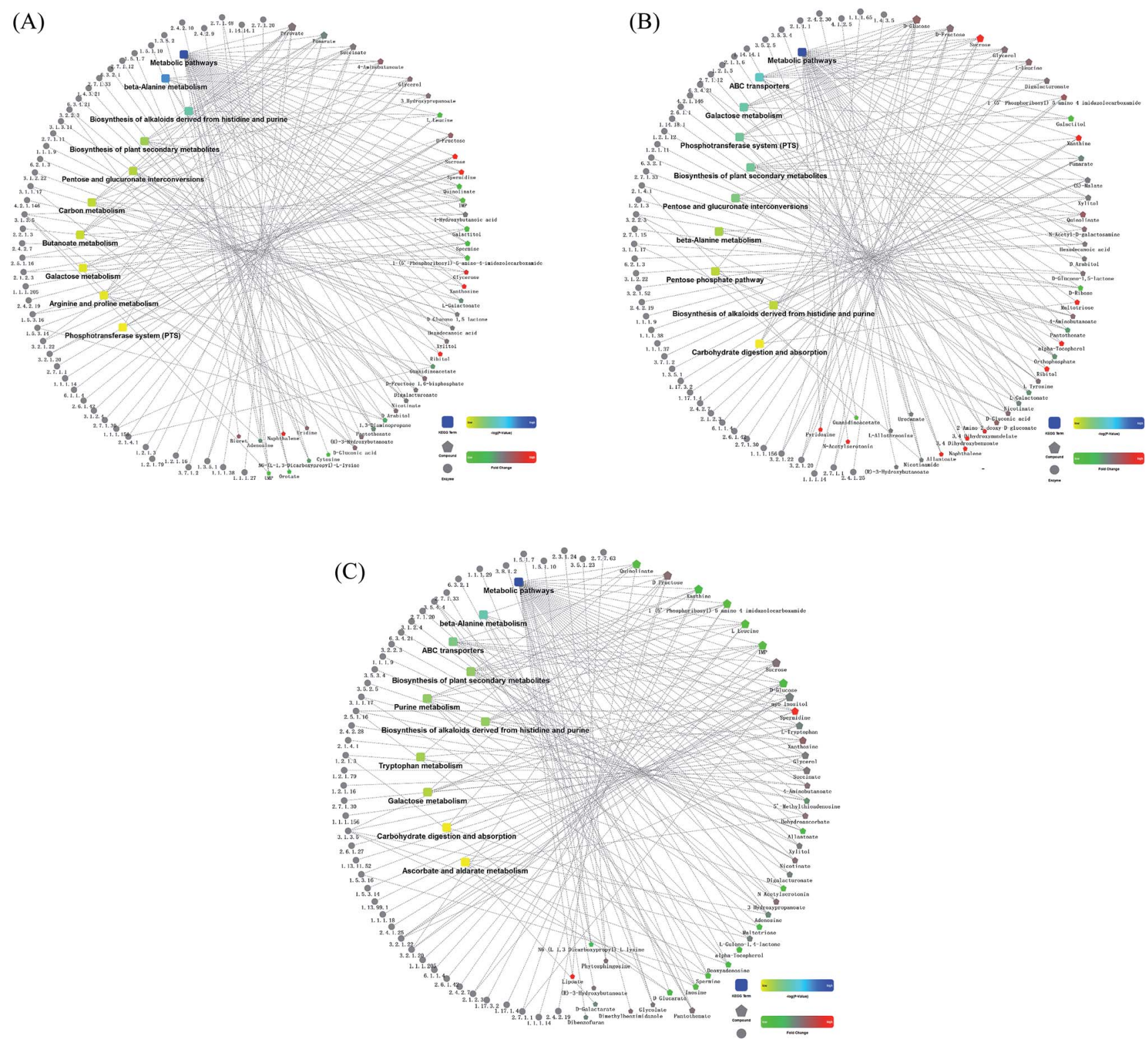

Fig. 5 Comparison analysis of metabolic network among three groups. The dotted line has been reported relevant verification. The KEGG pathway represented the molecular interaction of enzyme (with EC number) and compound. Red represented up-regulation, green represented down-regulation, yellow and blue represented significant changes from low to high. (A) The comparison between TG and RG groups. (B) The comparison between TG and NG groups. (C) The comparison between RG and NG groups. TG, Cordyceps militaris (L.) Link cultured on Tussah silkmoth pupae. RG, Cordyceps militaris (L.) Link cultured on rice. NG, Cordyceps sinensis.

(C18.1N9C) was highest, followed by linoleic acid (C18.2N6C). Relative contents of unsaturated fatty acid were highest in TG (almost $80 \%$ of fatty acids), middle in RG (55\% of fatty acids) and lowest in NG (49\% of fatty acids). The results suggested that Tussah silkmoth pupae improved the contents of unsaturated fatty acids of $C$. militaris (L.) Link when compared with the RG group.

\subsection{Cordycepin identification}

The contents of cordycepin reached $1863 \mathrm{mg} \mathrm{kg}^{-1}$ and $105 \mathrm{mg} \mathrm{g}^{-1}$ of the extracts of $C$. militaris (L.) Link cultured on Tussah silkmoth pupae. The cordycepin sample purified by this process was $92.6 \%$ pure compared to the cordycepin standard (Fig. 6A and B). The cordycepin standard and the purified sample were scanned in the infrared spectrum of $4000-400 \mathrm{~cm}^{-1}$ respectively. The spectral characteristics of the infrared spectrum of the cordycepin standard and the purified sample were consistent, and the characteristic spectra were the same (Fig. 6C and D).

\subsection{Anti-breast-tumor activity}

MCF-7 cells were observed under an inverted microscope: cells grew well, spindle-shaped and uniform size with high-level cell division in CG, NG and RG groups (Fig. 7A-C). The cells grew slowly in TG and LG Groups (Fig. 7D and E). Cell growth was significantly inhibited, cells become rounded, cell membranes became wide, cell contents were darken, and cell shape became irregular in HG group (Fig. 7F). The cellular structure of MCF-7 
Table 2 The contents of fatty acid $\left(n=3, \mathrm{mg} \mathrm{kg}^{-1}\right)^{a}$

\begin{tabular}{lrrr}
\hline Fatty acids & \multicolumn{2}{l}{ TG } & \multicolumn{1}{c}{ RG } \\
\hline C14.0 & 39.08 & 68.10 & 68.00 \\
C15.0 & 213.28 & 499.10 & 70.70 \\
C16.0 & 11594.65 & 12299.25 & 14096.40 \\
C16.1 & 379.45 & 444.58 & 966.45 \\
C17.0 & 417.88 & 1495.50 & 174.33 \\
C17.1 & 74.43 & 392.08 & 73.55 \\
C18.0 & 4007.95 & 4418.90 & 2479.03 \\
C18.1N9C & 36859.00 & 1630.45 & 2188.40 \\
C18.2N6C & 25225.45 & 20308.55 & 12589.50 \\
C20.0 & 26.88 & 37.90 & 40.33 \\
C20.1 & 18.43 & 32.05 & 121.50 \\
C20.2 & 17.88 & 49.10 & 196.63 \\
C21.0 & 24.63 & 29.90 & 10.18 \\
C22.0 & 42.63 & 56.48 & 24.83 \\
C23.0 & 207.05 & 152.90 & 18.03 \\
C24.0 & 24.10 & 27.88 & 16.05 \\
Total amount & 79172.73 & 41942.70 & 33133.88 \\
Saturated fatty acid & 16598.10 & 19085.90 & 16997.85 \\
Unsaturated fatty acid & 62574.63 & 22856.80 & 16136.03 \\
Saturated fatty acid ratio\% & 20.96 & 45.50 & 51.30 \\
Unsaturated fatty acid\% & 79.04 & 54.50 & 48.70 \\
Linoleic acid\% & 31.86 & 48.42 & 38.00 \\
Oleic acid\% & 46.56 & 3.89 & 6.60 \\
Palmitic acid\% & 14.64 & 29.32 & 42.54 \\
Stearic acid\% & 5.06 & 10.54 & 7.48 \\
a $\%$ & & &
\end{tabular}

${ }^{a}$ TG, Cordyceps militaris (L.) Link cultured on Tussah silkmoth pupae. RG, Cordyceps militaris (L.) Link cultured on rice. NG, Cordyceps sinensis.

was observed under transmission electron microscope. The cell membrane was intact, and the nucleus and organelle structure were intact (Fig. 8A-C). The nuclear chromatin edge of MCF-7 cells in the treatment group was concentrated, and the endoplasmic reticulum became loose. Vacuoles was fused with the membrane, and apoptotic cells were with typical crescent nucleus, nuclear pyknosis and intact membrane but foaming (Fig. 8D). The nuclear chromatin edge of MCF-7 cells was concentrated, the cytoplasm was concentrated, and the endoplasmic reticulum became loose (Fig. 8E). The nuclear chromatin of the cells aggregated to the periphery, the cytoplasm was concentrated, the endoplasmic reticulum became loose, and fused with the membrane to form vacuoles (Fig. 8F).

MTT assay showed that cordycepin exerted inhibitory function for the growth of MCF-7. With the increase in the concentration of cordycepin, the growth rate of MCF-7 was reduced (Fig. 9, $P<0.05$ ). Thus, cordycepin inhibited the growth of MCF-7 cells in a dose-depend way.

In this experiment, the anti-tumor effect of different groups on tumor-bearing mice was observed by establishing a model of S180 tumor-bearing mice, and the anti-tumor pharmacological effects of different groups were studied. TG showed anti-tumor activities when compared with other groups (CG, NG and RG, $P$ $<0.05$, Fig. 10). $30 \mathrm{mg} \mathrm{kg}{ }^{-1}$ cordycepin had a good anti-tumor effect on breast-tumor models. With the increase of dosage, anti-tumor effect was enhanced (Fig. 10). The inhibitory rate reached the highest levels with more than $60 \%$ in the HG group when compared with the CG group (Fig. 10).

\section{Discussion}

Cordyceps species is a medicinal fungus with anti-tumor, antioxidant, anti-aging, anti-radiation, antibacterial and antiviral properties with few side effects. Meanwhile, it can improve
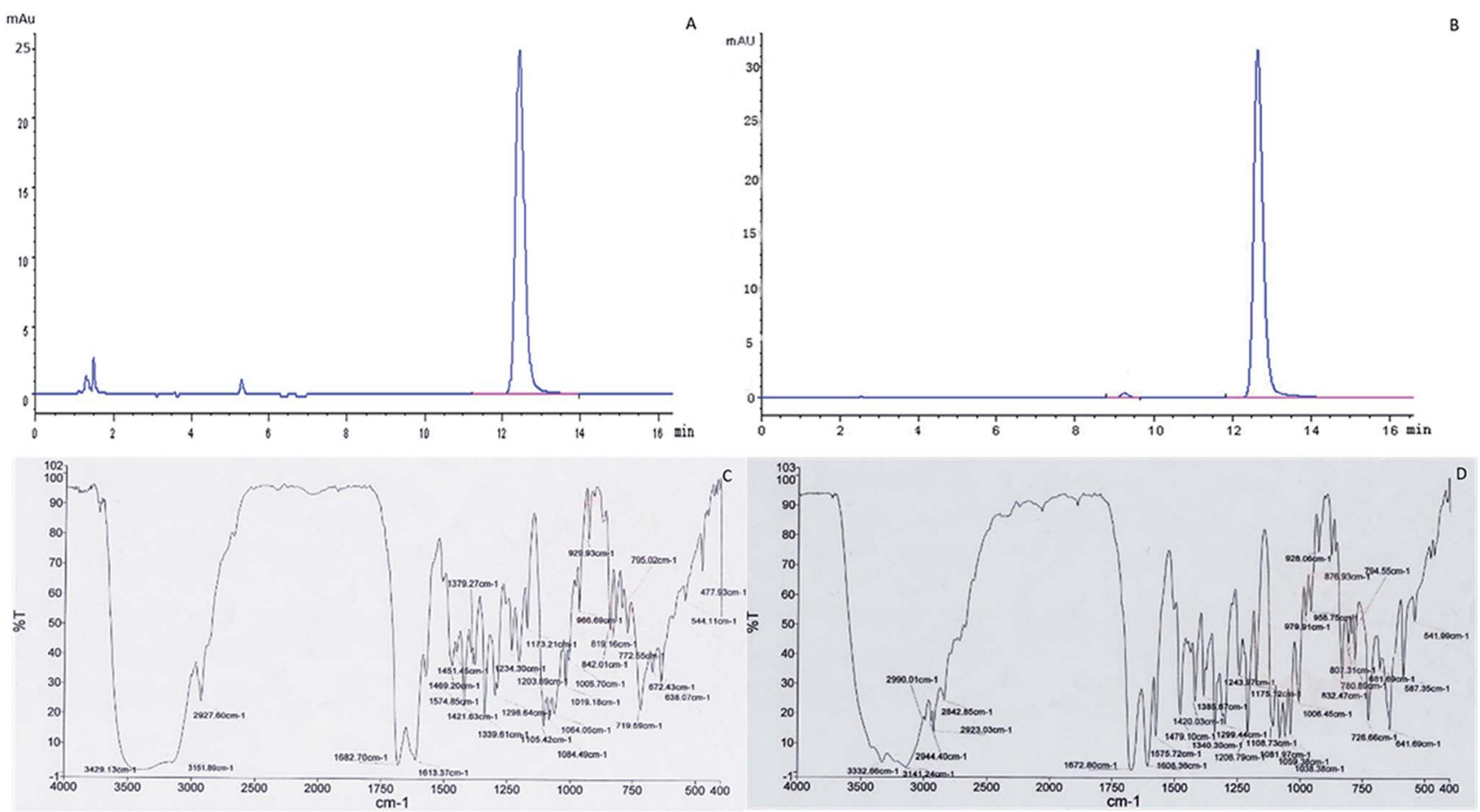

Fig. 6 Analysis of purified cordycepin. (A) HPLC analysis of purified cordycepin. (B) HPLC analysis of cordycepin standard. (C) Transform infrared spectroscopy (FTIR) spectra analysis of purified cordycepin. (D) FTIR spectra analysis of cordycepin standard. 


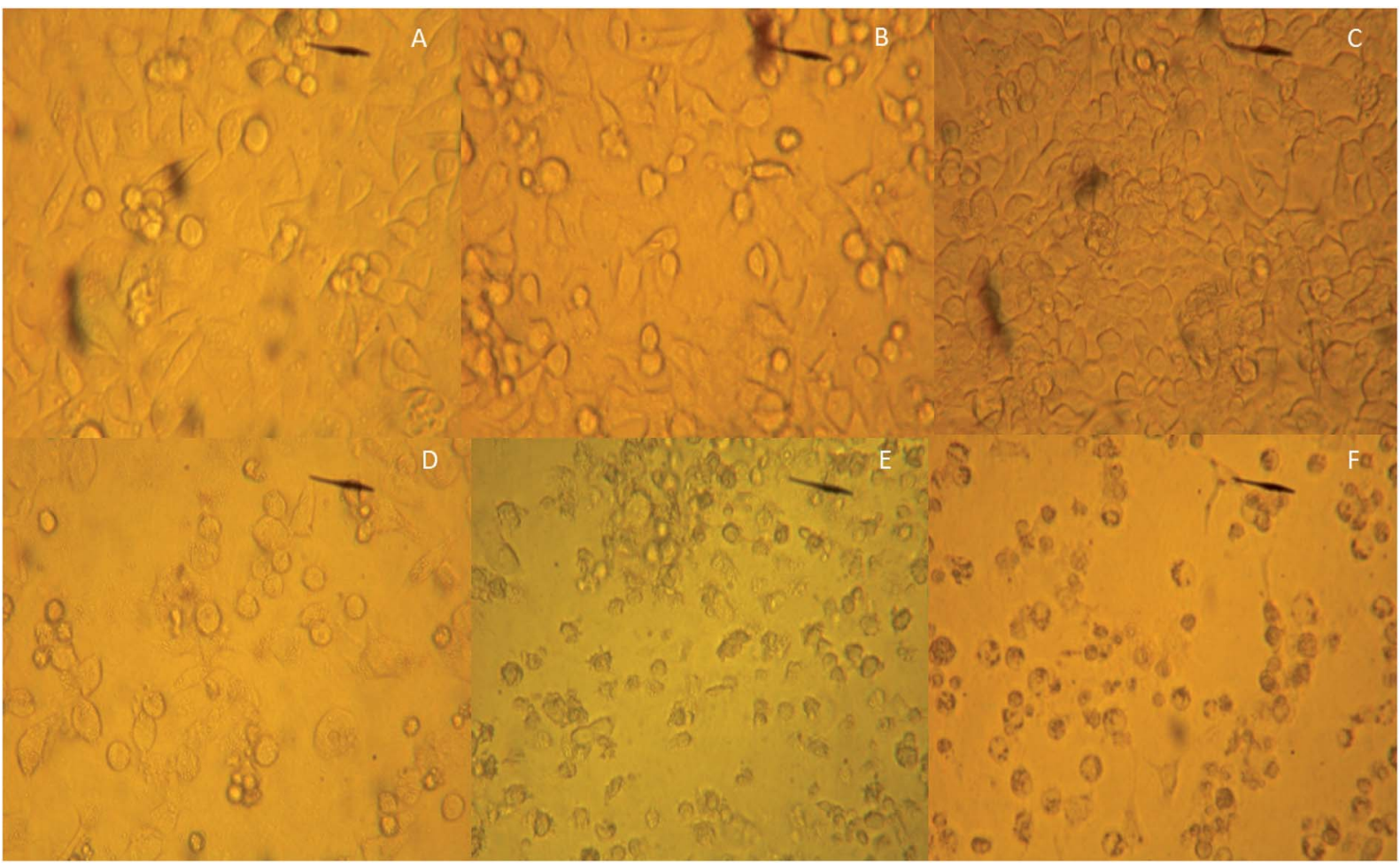

Fig. 7 The comparison of the effects of different groups on the cellular morphology of breast cancer cell line MCF-7. (A) The control group of MCF-7 cells. (B) The effects of C. sinensis on MCF-7 cells. (C) The effects of Cordyceps militaris (L.) Link cultured with rice on MCF-7 cells. (D) The effects of Cordyceps militaris (L.) Link cultured with Tussah silkmoth pupae on MCF-7 cells. (E) The effects of $30 \mathrm{mg} \mathrm{kg}^{-1}$ cordycepin on MCF-7 cells. (F) The effects of $90 \mathrm{mg} \mathrm{kg}^{-1}$ cordycepin on MCF-7 cells.

immune system, nervous system, cardiovascular system, respiratory system and renal function. C. militaris (L.) Link is a perfect substitute for wild Cordyceps sinensis with potential pharmaceutical values.

3-Deoxygenadenosine (cordycepin), hesperitin, and 3hydroxyflavone were up-regulated in the TG group (Fig. 4). 3Deoxygenadenosine is an active component of C. militaris, which can improve cardiovascular and has been identified to have anti-metastatic effect on tumor progression..$^{15}$ Cordycepin has been reported to improve the efficacy of conventional chemotherapy for Epstein-Barr-virus-positive tumors. ${ }^{16}$ Cordycepin is a major anti-tumor agent in C. militaris ${ }^{7}$ and was found to be increased on the medium with Tussah silkmoth pupae in the present study. The results provided a simple method to improve the contents of cordycepin in C. militaris. The present findings suggested that Tussah silkmoth pupae may increase anti-breast-tumor properties of C. militaris via its major metabolite cordycepin (Fig. 8 and 9).

Hesperitin is a compound derived from hesperidin, which is a natural flavanone glycoside that has anti-inflammatory, antioxidant and anti-angiogenic activities ${ }^{17}$. Flavonoids are naturally occurring phenolic compounds. Among them, 3-hydroxyflavone contains a 3-hydroxyl group with its 4-keto group and double bond between 2- and 3-carbon atoms, has multiple pharmacological properties, including antiviral, antitumor, anti-inflammatory and antioxidant activities. ${ }^{18}$ Tussah silkmoth pupae induced biosynthesis of unsaturated fatty acids (Table
S1†), which may be beneficial for preventing breast cancer. ${ }^{19}$ Unsaturated fatty acids can promote the multitarget retinoic acid activity against human breast cancer cells. ${ }^{19}$ The contents of oleic acid were highest in the TG group. Oleic acid had a protective role against progression of pancreatic ductal adenocarcinoma in those with higher body mass index possibly through influencing hyperinsulinaemia. ${ }^{20}$ Metabolic network analysis showed that Tussah silkmoth pupae induced metabolites pathways involving with the upregulation of sucrose, spermidine, glycerone, xanthosine, ribitol and naphthalene. An increase in the uptake of the spermidine has been found to be related to the decrease in overall and cancer-specific mortality. ${ }^{21}$ Naphthalene derivatives had higher toxicity towards cancer cell lines and may be a potential anti-cancer lead molecule, especially for breast cancers. ${ }^{22}$

Lactic acid was more than 4-fold upregulated in the TG group when compared with the RG and NG groups. The acid suppresses the uptake of $18 \mathrm{~F}$-fluorodeoxyglucose in the microenvironment of tumor cells. ${ }^{23}$ Lactic acid has been reported to increase the cytotoxicity of natural killer cells to myelogenous leukemia and colorectal tumor cells. ${ }^{24}$ Aminobutyric acid was more than 5-fold and 10-fold upregulated in TG when compared with RG and CG groups, respectively. Gamma-aminobutyric acid has been found to exert anti-tumor properties and regulate tumor growth by affecting JNKs signaling pathway. ${ }^{25}$ Succinic acid was more than two-fold upregulated in TG when compared with two other groups. The acid has been found to 


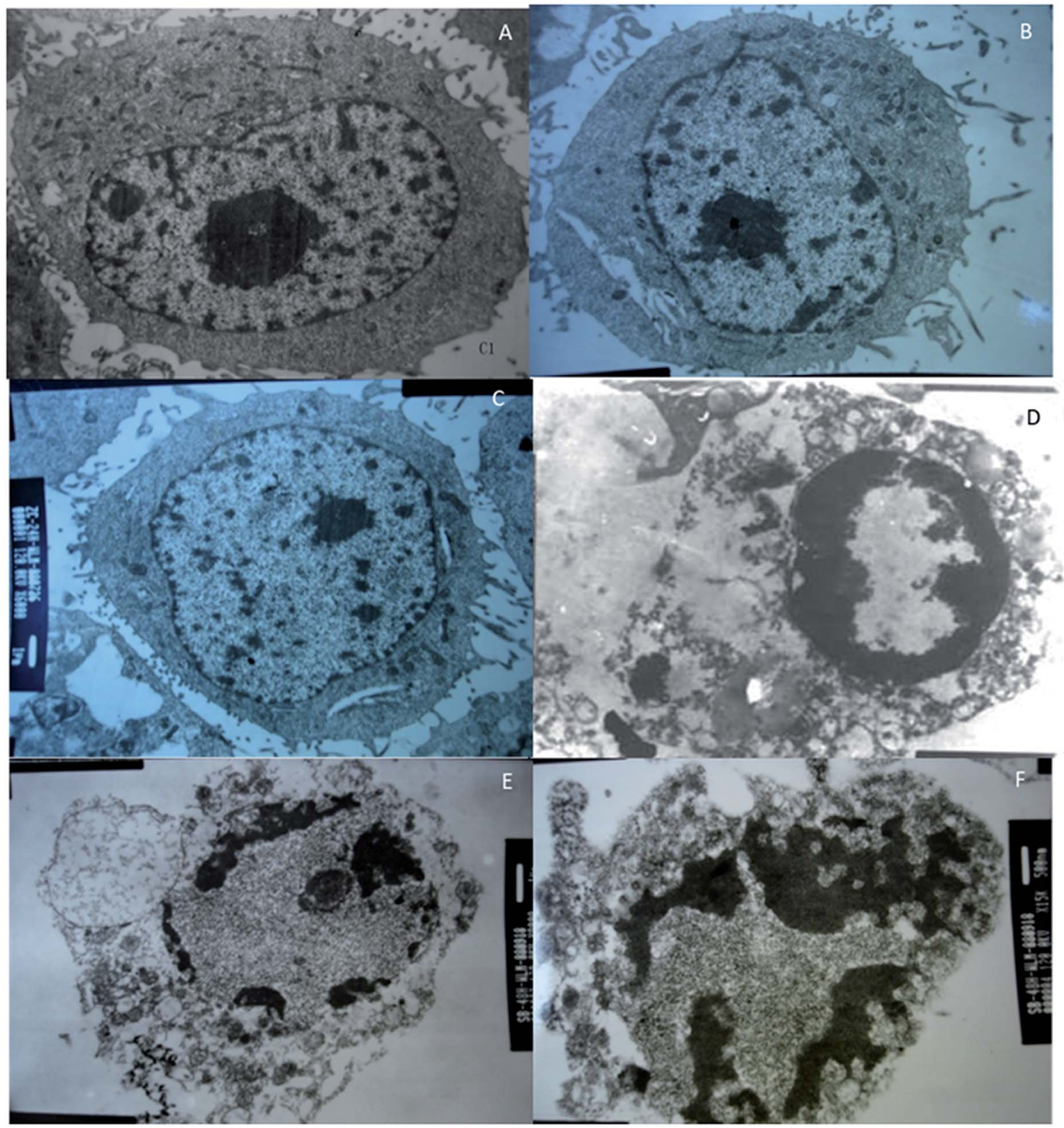

Fig. 8 Transmission electron microscopy (TEM) analysis of the effects of different groups on the cellular structure of breast cancer cell line MCF-7. (A) The control group of MCF-7 cells. (B) The effects of C. sinensis on MCF-7 cells. (C) The effects of Cordyceps militaris (L.) Link cultured with rice on MCF-7 cells. (D) The effects of Cordyceps militaris (L.) Link cultured with Tussah silkmoth pupae on MCF-7 cells. (E) The effects of 30 mg kg ${ }^{-1}$ cordycepin on MCF-7 cells. (F) The effects of $90 \mathrm{mg} \mathrm{kg}^{-1}$ cordycepin on MCF-7 cells.

show inhibitory function for tumor cells. ${ }^{26}$ Gallic acid was more than two-fold and three-fold upregulated in TG when compared with RG and NG groups, respectively. The natural phenol can induce apoptosis in human lung cancer cell lines. ${ }^{27}$ All the results suggest that Tussah silkmoth pupae may improve antibreast-tumor properties of $C$. militaris.

Carnitine and alpha-tocopherol were upregulated in the RG group (Fig. 4). Carnitine has been regarded as to reduce plasma levels of lipids and has antioxidant potential. ${ }^{28}$ Alphatocopherol has the potential for preventing and treating of oxidative- or inflammatory diseases. ${ }^{29}$ Gentiobiose and tartaric acid were up-regulated in the NG group (Fig. 4). Gentiobiose has been reported to have anti-fatigue and vasoprotective effects and is potential agent for controlling fatigue- and related syndrome. ${ }^{30}$ Tartaric acid has been widely used in the pharmaceutical industry but scarcely exists in nature. ${ }^{31}$

There were some limitations in the present work. Other components and their function were not compared among TG, RG and CG groups although the metabolomics of Cordyceps fungi was performed. Small size of an animal population may lead to cases of bias. Metabolomics analyses and network analysis provided large data in this paper, but additional 


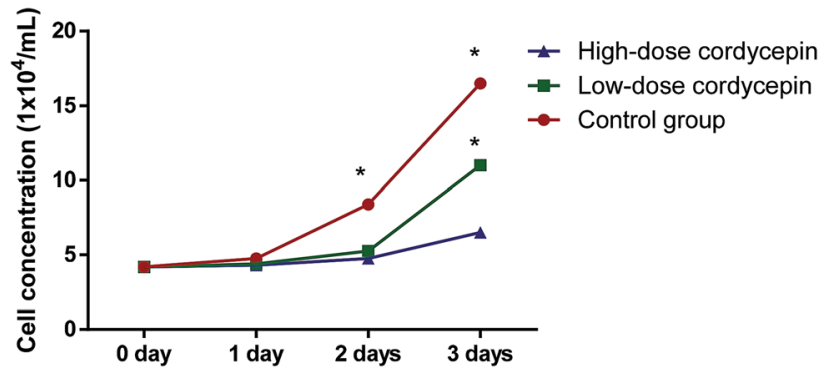

Fig. 9 The effects of cordycepin on the growth of MCF-7. Low-dose, $30 \mu \mathrm{g} \mathrm{mL}^{-1}$ cordycepin and high-dose, $90 \mu \mathrm{g} \mathrm{mL}^{-1}$ cordycepin. $* P<$ 0.05 vs. a control group.
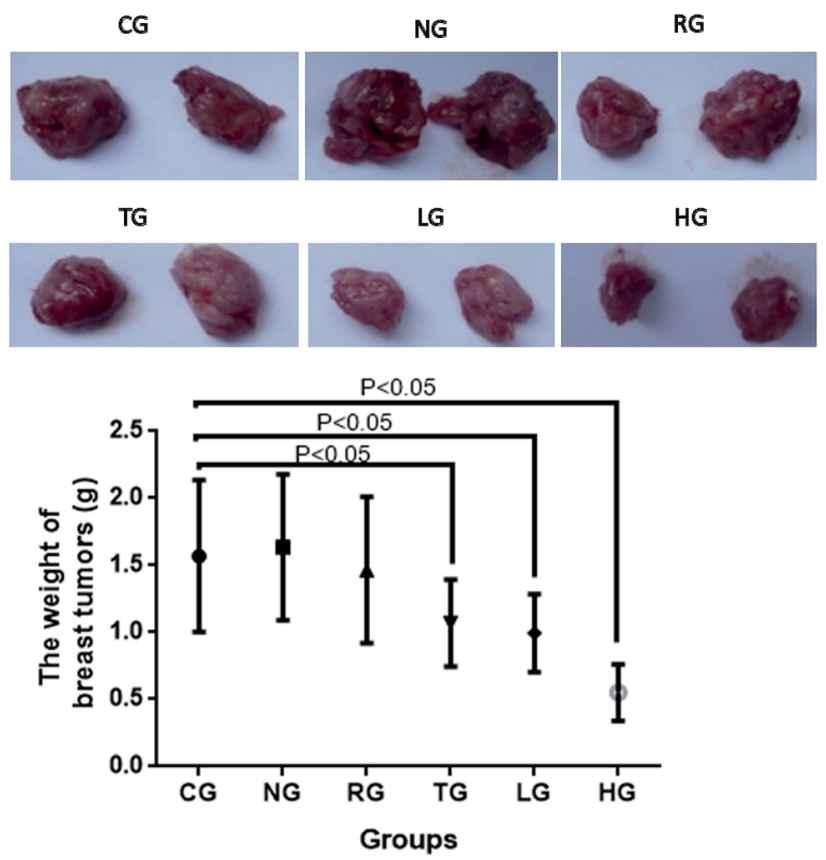

Fig. 10 Anti-breast-tumor effect of different groups on tumorbearing mice. Tumor-bearing mice control group, $20 \mu \mathrm{L}(\mathrm{kg} \mathrm{d})^{-1}$ normal saline (CG group); $200 \mathrm{mg}\left(\mathrm{kg}^{-1} \mathrm{~d}\right)$ extracts of natural Cordyceps sinensis (NG group); $200 \mathrm{mg}(\mathrm{kg} \mathrm{d})^{-1}$ extracts of Cordyceps militaris (L.) Link cultured on rice (RG group); $200 \mathrm{mg}(\mathrm{kg} \mathrm{d})^{-1}$ extracts of Cordyceps militaris (L.) Link cultured on Tussah silkmoth pupae (TG group); the extract of low-dose cordycepin $30 \mathrm{mg}$ (kg d) ${ }^{-1}$ (LG group); and high-dose cordycepin $90 \mathrm{mg}(\mathrm{kg} \mathrm{d})^{-1}$ (HG group).

experiments are still highly demanded to confirm the conclusion. Further work should be performed to confirm the potential valuable components among three groups in the future.

\section{Conclusions}

At present, the research on the metabolomics of Cordyceps fungi is still less, ${ }^{32}$ and more focuses on the optimization of the known active ingredient content, function and culture conditions. Metabolomics analysis provides more comprehensive information for the biological metabolism of Cordyceps species. Therefore, metabolomics of C. militaris (L.) Link and Cordyceps sinensis can help us to better understand and utilize the resources of $C$. militaris (L.) Link and optimize the culture medium. Tussah silkmoth pupae improved anti-breast-tumor properties of $C$. militaris via its major metabolite cordycepin in an animal model. However, the research on metabolomics is still in the initial stage and the functions of many metabolites remain unknown. Therefore, further work is highly demanded to identify various metabolites and construct metabolite databases.

\section{Conflicts of interest}

There are no conflicts to declare.

\section{Reference}

1 C. F. Kuo, C. C. Chen, C. F. Lin, M. S. Jan, R. Y. Huang, Y. H. Luo, et al., Abrogation of streptococcal pyrogenic exotoxin B-mediated suppression of phagocytosis in U937 cells by Cordyceps sinensis mycelium via production of cytokines, Food Chem. Toxicol., 2007, 45(2), 278-285.

2 E. Kubo, N. Yoshikawa, M. Kunitomo, S. Kagota, K. Shinozuka and K. Nakamura, Inhibitory effect of Cordyceps sinensis on experimental hepatic metastasis of melanoma by suppressing tumor cell invasion, Anticancer Res., 2010, 30(9), 3429-3433.

3 Y. Peng, K. Huang, L. Shen, Y. Y. Tao and C. H. Liu, Cultured Mycelium Cordyceps sinensis allevi notates CCl4-induced liver inflammation and fibrosis in mice by activating hepatic natural killer cells, Acta Pharmacol. Sin., 2016, 37(2), 204-216.

4 N. Wang, J. Li, X. Huang, W. Chen and Y. Chen, Herbal Medicine Cordyceps sinensis Improves Health-Related Quality of Life in Moderate-to-Severe Asthma, J. EvidenceBased Complementary Altern. Med., 2016, 2016, 6134593.

5 Y. Xiao, Q. Huang, Z. Zheng, H. Guan and S. Liu, Construction of a Cordyceps sinensis exopolysaccharideconjugated selenium nanoparticles and enhancement of their antioxidant activities, Int. J. Biol. Macromol., 2017, 99, 483-491.

6 A. Bizarro, I. C. Ferreira, M. Sokovic, L. J. van Griensven, D. Sousa, M. H. Vasconcelos, et al., Cordyceps militaris (L.) Link Fruiting Body Reduces the Growth of a Non-Small Cell Lung Cancer Cell Line by Increasing Cellular Levels of p53 and p21, Molecules, 2015, 20(8), 13927-13940.

7 T. Wada, I. W. Sumardika, S. Saito, I. M. W. Ruma, E. Kondo, M. Shibukawa, et al., Identification of a novel component leading to anti-tumor activity besides the major ingredient cordycepin in Cordyceps militaris extract, J. Chromatogr. B: Anal. Technol. Biomed. Life Sci., 2017, 1061-1062, 209-219.

$8 \mathrm{R}$. Chen and M. Ichida, Infection of the silkworm, Bombyx mori, with Cordyceps militaris, J. Insect Biotechnol. Sericol., 2002, 71(1), 61-63.

9 L. X. Guo, X. M. Xu, F. R. Liang, J. P. Yuan, J. Peng, C. F. Wu, et al., Morphological Observations and Fatty Acid Composition of Indoor-Cultivated Cordyceps sinensis at 
a High-Altitude Laboratory on Sejila Mountain, Tibet, PLoS One, 2015, 10(5), e0126095.

$10 \mathrm{~J}$. Boccard and D. N. Rutledge, A consensus orthogonal partial least squares discriminant analysis (OPLS-DA) strategy for multiblock Omics data fusion, Anal. Chim. Acta, 2013, 769, 30-39.

11 D. Szklarczyk, J. H. Morris, H. Cook, M. Kuhn, S. Wyder, M. Simonovic, et al., The STRING database in 2017: quality-controlled protein-protein association networks, made broadly accessible, Nucleic Acids Res., 2017, 45(D1), D362-D368.

12 A. V. Antonov, E. E. Schmidt, S. Dietmann, M. Krestyaninova and $\mathrm{H}$. Hermjakob, R spider: a network-based analysis of gene lists by combining signaling and metabolic pathways from Reactome and KEGG databases, Nucleic Acids Res., 2010, 38, W78-W83.

13 M. Kashif, Y. Hwang, G. Hong and G. Kim, In vitro comparative cytotoxic effect of Nimbolide: A limonoid from Azadirachta indica (Neem tree) on cancer cell lines and normal cell lines through MTT assay, Pak. J. Pharm. Sci., 2017, 30(suppl. 3), 967-973.

14 P. Imesch, A. Goerens, D. Fink and A. Fedier, MLH1-deficient HCT116 colon tumor cells exhibit resistance to the cytostatic and cytotoxic effect of the poly(A) polymerase inhibitor cordycepin (3'-deoxyadenosine) in vitro, Oncol. Lett., 2012, 3(2), 441-444.

15 P. Zhang, C. Huang, C. Fu, Y. Tian, Y. Hu, B. Wang, et al., Cordycepin (3'-deoxyadenosine) suppressed HMGA2, Twist1 and ZEB1-dependent melanoma invasion and metastasis by targeting miR-33b, OncoTargets Ther., 2015, 6(12), 9834-9853.

16 Y. Du, J. Yu, L. Du, J. Tang and W. H. Feng, Cordycepin enhances Epstein-Barr virus lytic infection and EpsteinBarr virus-positive tumor treatment efficacy by doxorubicin, Cancer Lett., 2016, 376(2), 240-248.

17 W. X. Li, X. Chen, Y. Yang, H. M. Huang, H. D. Li, C. Huang, et al., Hesperitin derivative-11 suppress hepatic stellate cell activation and proliferation by targeting PTEN/AKT pathway, Toxicology, 2017, 381, 75-86.

18 B. Butun, G. Topcu and T. Ozturk, Recent Advances on 3Hydroxyflavone Derivatives: Structures and Properties, Mini-Rev. Med. Chem., 2018, 18(2), 98-103.

19 P. L. Quiroga, E. A. Soria, M. A. Valentich and A. R. Eynard, Differential Potentiation of Retinoic Acid Effects against Human Breast Cancer Cells by Unsaturated Fatty Acids, Nutr. Cancer, 2018, 1-8.

20 P. J. Banim, R. Luben, K. T. Khaw and A. R. Hart, Dietary oleic acid is inversely associated with pancreatic cancer Data from food diaries in a cohort study, Pancreatology, 2018, 18(6), 655-660.
21 F. Pietrocola, F. Castoldi, O. Kepp, D. Carmona-Gutierrez, F. Madeo and G. Kroemer, Spermidine reduces cancerrelated mortality in humans, Autophagy, 2018, 1-4.

22 P. R. Kavitha Rani, Y. S. Mary, A. Fernandez, S. Anu Priya, Y. S. Mary and R. Thomas, Single crystal XRD, DFT investigations and molecular docking study of 2-((1,5dimethyl-3-oxo-2-phenyl-2,3-dihydro-1H-pyrazol-4-yl)amino) naphthalene-1,4-D-ione as a potential anti-cancer lead molecule, Comput. Biol. Chem., 2018, 78, 153-164.

23 S. Turkcan, L. Kiru, D. J. Naczynski, L. S. Sasportas and G. Pratx, Lactic acid accumulation in the tumor microenvironment suppresses 18F-FDG uptake, Cancer Res., 2019, 79, 410-419.

24 T. Yamane, T. Sakamoto, T. Nakagaki and Y. Nakano, Lactic Acid Bacteria from Kefir Increase Cytotoxicity of Natural Killer Cells to Tumor Cells, Foods, 2018, 7(4), 1-9.

25 H. Tian, J. X. Wu, F. X. Shan, S. N. Zhang, Q. Cheng, J. N. Zheng, et al., Gamma-aminobutyric acid induces tumor cells apoptosis via GABABR1.beta-arrestins.JNKs signaling module, Cell Biochem. Biophys., 2015, 71(2), 679688.

26 N. Nakamura, A. Hirakawa, J. J. Gao, H. Kakuda, M. Shiro, Y. Komatsu, et al., Five new maleic and succinic acid derivatives from the mycelium of Antrodia camphorata and their cytotoxic effects on LLC tumor cell line, J. Nat. Prod., 2004, 67(1), 46-48.

27 M. Kawada, Y. Ohno, Y. Ri, T. Ikoma, H. Yuugetu, T. Asai, et al., Anti-tumor effect of gallic acid on LL-2 lung cancer cells transplanted in mice, Anticancer Drugs, 2001, 12(10), 847-852.

28 M. Ramazani, D. Qujeq and Z. Moazezi, Assessing the Levels of L-Carnitine and Total Antioxidant Capacity in Adults With Newly Diagnosed and Long-Standing Type 2 Diabetes, Can. J. Diabetes, 2019, 43(1), 46-50.

29 X. Yan, Y. Liu, T. Xie and F. Liu, alpha-Tocopherol protected against cobalt nanoparticles and $\operatorname{cocl} 2$ induced cytotoxicity and inflammation in Balb/3T3 cells, Immunopharmacol. Immunotoxicol., 2018, 40(2), 179-185.

30 Y. Lin, H. L. Liu, J. Fang, C. H. Yu, Y. K. Xiong and K. Yuan, Anti-fatigue and vasoprotective effects of quercetin-3-Ogentiobiose on oxidative stress and vascular endothelial dysfunction induced by endurance swimming in rats, Food Chem. Toxicol., 2014, 68, 290-296.

31 W. Dong, F. Zhao, F. Xin, A. He, Y. Zhang, H. Wu, et al., Ultrasound-assisted d-tartaric acid whole-cell bioconversion by recombinant Escherichia coli, Ultrason. Sonochem., 2018, 42, 11-17.

32 J. N. Choi, J. Kim, M. Y. Lee, D. K. Park, Y. S. Hong and C. H. Lee, Metabolomics revealed novel isoflavones and optimal cultivation time of Cordyceps militaris fermentation, J. Agric. Food Chem., 2010, 58(7), 4258-4267. 